\title{
3 Research Square

\section{Early detection of SARS-CoV-2 P.1 variant in Southern Brazil and reinfection of the same patient by P.2}

Mariana Soares da Silva ( $\square$ marisoares.vet@hotmail.com )

Universidade Feevale

Meriane Demoliner

Universidade Feevale

Alana Witt Hansen

Universidade Feevale

Juliana Schons Gularte

Universidade Feevale

Flávio Silveira

Universidade Feevale

Fágner Henrique Heldt

Universidade Feevale

Micheli Filippi

Universidade Feevale

Francini Pereira da Silva

Universidade Feevale

Larissa Mallmann

Universidade Feevale

Pietra Fink

Universidade Feevale

Luana Letícia da Silva

Secretaria Municipal da Saúde de Campo Bom

Matheus Nunes Weber

Universidade Feevale

Paula Rodrigues de Almeida

Universidade Feevale

Juliane Deise Fleck

Universidade Feevale

Fernando Rosado Spilki ( $\square$ fernandors@feevale.br )

Universidade Feevale 


\section{Research Article}

Keywords: COVID-19, reinfection, P.1 lineage, SARS-CoV-2, Brazil

Posted Date: April 21st, 2021

DOI: https://doi.org/10.21203/rs.3.rs-435535/v2

License: (c) (i) This work is licensed under a Creative Commons Attribution 4.0 International License. Read Full License 


\section{Abstract}

Multiple variants of Severe acute respiratory syndrome coronavirus 2 (SARS-CoV-2) are constantly reported across the world. B.1.1.28 lineage has been evolving in Brazil since February 2020 and originated P.1 (VOC) and P.2 (VOI). Here we describe an early case of P.1 primary infection in Southern Brazil in late November 2020, soon after the emergence of the variant in Manaus, Northern Brazil. The same patient suffered a reinfection case by another B1.1.28 variant, namely P.2, in March, 2021. Genomic analysis showed genetically significant differences between the viruses recovered in both infections. P.1 lineage at the first episode and P.2 at the reinfection. We also have investigated the circulation of P.1 in the same region by differential RT-qPCR, showing that this was an isolated case of P.1 at the time of detection, whereas the variant disseminated and became prominent from late January 2021 to the end of March 2021.

\section{Introduction}

Emergence of novel SARS-CoV-2 lineages is currently a worldwide concern. Continuous variant rising is probably related to increased virus transmission rates and immune escape. Lately, research groups have reported the emergence of multiple variants of concern (VOC) or interest (VOI), such as P.1 [1], P.2 [2], N.9 [3] N.10 [4] and B.1.351 [5] in specific Brazilian regions. P.1 (VOC) and P.2 (VOI) variants have evolved from lineage B.1.1.28 [2, 6] and harbor E484K (P.1 and P.2) and N501Y (P.1) mutations, which are associated with increased transmissibility or immune evasion $[2,7]$. Protective immunity persistence caused by coronavirus disease 2019 (COVID-19) or vaccination are not yet well established and some reinfection cases have been reported [8]. In this study we report a reinfection SARS-CoV-2 case. The COVID-19 episodes occurred in a 39-year-old individual from Rio Grande do Sul, Southern Brazil. P.1 variant is of particular concern in the case of Brazil; the variant is widely spread in the country, being associated with local outbreaks of great magnitude due to an enhanced transmissibility. A recent study showed P1. more likely appeared in Manaus, Northern Brazil, in mid-November [9]. The first cases described attributed to P.1 in Southern Brazil so far are from the last week of January 2021.

In this post, we report the finding of a P.1 infection in a patient from Campo Bom, Rio Grande do Sul (Figure 1), the Southernmost state of Brazil in late November 2020. The same patient got infected by another variant, P.2 lineage in March 2021. We have also investigated further the circulation of P.1 in the same geographic region from the time of the first infection to the March 2021 using a differential VOC RTqPCR, and the results are presented as follows.

\section{Results}

\section{Case description and epidemiological findings}

A 39-years-old male patient with chronic cardiovascular disease and diabetes mellitus as history of comorbidities reported two clinical episodes of COVID-19. The first one was on November $30^{\text {th }}, 2020$, 
and the second on March $11^{\text {th }}, 2021$. During the first infection case, the symptoms and clinical signs of the patient had not been reported. However, the patient reported having contact with his brother, who tested positive for SARS-CoV-2 previously. He also visited his father at the hospital in a room shared with other COVID-19 diagnosed patients. In the second episode the patient had dyspnea, fatigue, and respiratory distress as symptoms; and saturation $<95 \%$ as a clinical sign. The second infection evolved with complications, and the patient was removed to an Intensive Care Unit (ICU) and intubated due to severe loss of pulmonary capacity. The patient unfortunately died on March $19^{\text {th }}, 2021$.

\section{Diagnostic laboratory findings}

Naso-oropharyngeal swab samples (November $30^{\text {th }}, 2020$ and March $11^{\text {th }}, 2021$ ) were received at Laboratório de Microbiologia Molecular of Universidade Feevale, Novo Hamburgo, RS, Brazil, for SARSCoV-2 diagnostics. Total nucleic acid was extracted with the commercial MagMAX ${ }^{\text {TM }}$ CORE Nucleic Acid Purification Kit (Applied biosystems ${ }^{\text {TM }}$ ) kit using the automated equipment KingFisher ${ }^{\text {TM }}$ Duo Prime (Thermo Fisher Scientific ${ }^{\mathrm{TM}}$ ). RT-qPCR for SARS-CoV-2 RNA detection targeting E gene was performed according to Charité protocols [10] and was carried out using AgPath-ID One-Step RT-PCR Reagents (Thermo Fisher Scientific ${ }^{\text {TM }}$ ). Both RT-qPCR assays were positive, showing a Ct value of 30.07 (LMM38991) and 18.83 (LMM50731). Additionally, a differential RT-qPCR for P1 lineage [11] that detects ORF 1b deletion (NSP6: S106 del, G107 del, F108 del), was performed. This deletion is a genetic signature of VOCs P.1, B.1.1.7, and B.1.351. The differential RT-qPCR was carried out using AgPath-ID One-Step RT-

PCR Reagents (Thermo Fisher Scientific ${ }^{\mathrm{TM}}$ ); primers and probes were used following the same protocol as described by Naveca et al. (2021) [11].

A total of 302 samples was tested with differential RT-qPCR for specific P.1 lineage detection (Ct values for E gene ranging between 10 and 20) randomly distributed over 18 weeks that started on November $30^{\text {th }}, 2020$ until April $4^{\text {th }}, 2021$. A total of 183 samples were negative and 119 were positive for P.1. Except for the reinfection patient (who was not analyzed due to the CT value), the circulation of P.1 in RS started was evidenced in our sampling from January $27^{\text {th }}, 2021$ (Figure 2a). Furthermore, the peak of P.1 lineage detections coincides with the peak of SARS-CoV-2 new cases (Figure 2b), hospitalizations (Figure 2c) and deaths (Figure 2d) in the neighboring region.

This study was approved by the National Committee of Research Ethics and the Institutional Ethical Review Board of the Universidade Feevale (protocol number: 33202820.7.1001.5348), following Brazilian regulations and international ethical standards.

\section{Genome sequencing and bioinformatic analysis}

Two high-quality SARS-CoV-2 whole-genome sequences, named LMM38991/2020 and LMM50731/2021 (EPI_ISL_1630158 and EPI_ISL_1629809) were recovered from the same patient for the first infection and reinfection, respectively. Whole genome library preparation was performed using QIAseq SARS-CoV-2 Primer Panel paired for library enrichment and QIAseq FX DNA Library UDI Kit, according to the 
manufacturer instructions (Qiagen, Hilden, Germany). The sequencing was implemented on an Illumina MiSeq platform using MiSeq Reagent Kit v3 (600-cycle). The FASTQ reads were imported to Geneious Prime, trimmed (BBDuk 37.25), and mapped against the reference sequence hCoV-

19/Wuhan/WIV04/2019 (EPI_ISL_402124) available in EpiCoV database from GISAID

(https://www.gisaid.org/ 45). Consensus sequences LMM38991/2020 and LMM50731/2021 presented a mean coverage of $1,405 x$ and $1,263 x$.

The sequences were first classified using the Pangolin COVID-19 Lineage Assigner tool (https://github.com/hCoV-2019/pangolin) [12] indicating the presence of two discordant SARS-CoV-2 lineages: P.1 lineage in the primo-infection (LMM38991/2020; Gisaid access EPI_ISL_EPI_ISL_1630158) and P.2 lineage (LMM50731/2021; Gisaid access EPI_ISL_1629809) at reinfection. In order to confirm the results, 97 Brazilian SARS-CoV-2 complete genomes and the reference sequence (EPI_ISL_402124) (>29 $\mathrm{kb})$ were retrieved from the GISAID database and aligned with the sequences generated herein. Sequence alignment was performed using Clustal Omega and the reference sequence from Wuhan was applied as outgroup. The Maximum Likelihood phylogenetic analysis under General Time Reversible model allowing for a proportion of invariable sites and substitution rates were inferred empirically in IQ-TREE v2.1.2 web server [13] applying 200 replicates and 1000 bootstrap and the analysis corroborated the previous results. LMM38991/2020 clearly clustered into P.1 lineage while LMM50731/2021 branched into P.2 group (Figure 3). LMM38991/2020 and LMM50731/2021 displayed the typical P.1 and P.2 spike protein E484K mutations and INDELs (Figure 4). LMM38991/2020 presented all 11 typical P.1 amino acid nonsynonymous changes in S protein (L18F, T20N, P26S, D138Y, R190S, K417T, E484K, N501Y, D614G, H655Y, T1027I, V1176F) and LMM50731/2021 presented three previously P.2 reported amino acid changes in S protein (E484K, D614G, V1176F) (Figure 4).

\section{Discussion And Conclusions}

This study described a P.1 lineage primo-infection followed by P.2 lineage reinfection in a three-month interval. A male, 39-years-old patient with comorbidities history, presented two clinical episodes of COVID19. Few reinfection cases were described worldwide, especially with different lineages detection $[14,15]$. Although, there are apparently more cases reporting that reinfection presents asymptomatic/mild disease [16], and some cases showed a more severe illness in the second episode $[15,17]$ as the case described here. These evidences are reinforced by the apparent low viral quantification by RT-qPCR in primoinfection. Virus immune escape or limited and transitory protective immunity might be the cause of this reinfection as observed in last cases to date [8], specially emerging lineages reinfections (P.1 and P.2) that might also reflect ability of S:K484 virus to escape from anti-SARS-CoV-2 neutralizing antibodies. It is noticeable that P.1 and P.2 variants share at the typical E484K mutation in the RBD site, which has been related to antibody escape in patients previously immunized by non-mutated lineages. Although the patient may have formed antibodies that included the S:484K site, it apparently did not prevent a P.2 secondary infection. 
P.1 lineage most probably emerged in Brazil in November 2020 in Manaus, Northern region [9, 11]. Our findings showed that P.1 lineage was present in Southern Brazil soon after, in late November. A recent evolutionary molecular clock study inferred that the virus surged in mid-November [9] approximately 15 days before our P.1 detection. According to our retrospective analysis, based on differential qRT_PCR, it's important to note that despite the case reported herein, P.1 lineage did not spread in this first moment in the neighboring region. Expressive number of positive P.1 cases were found only at the end of January in this region and rapidly replaced the parental lineages.

In summary, this study reported and characterized an early case of P.1 primary infection in a reinfection episode in Southern, Brazil. P.1 lineage is spreading rapidly across Brazil [18] and after establishing is related with exponentially increasing transmissibility and hospitalization rate [9] as observed in our date. It's important to understand the new lineages origin but since the patient made several personal contacts, including with close family members also infected with SARS-CoV-2; which may denote a probable situation of higher risk of infection. Despite our efforts, due the high number of infected contacts the individual had in the first infection, it was impossible to establish the infection source.

\section{Declarations}

\section{Acknowledgements}

This work is an initiative of Rede Corona-ômica BR MCTI/FINEP affiliated to RedeVírus/MCTI (FINEP = 01.20.0029.000462/20, CNPq = 404096/2020-4).

\section{Conflict of Interest}

The authors have no conflicts of interest to declare.

\section{References}

1. Candido S, Mishra S, Crispim MAE, et al (2021) Genomics and epidemiology of a novel SARS-CoV-2 lineage in Manaus, Brazil. medRxiv. https://doi.org/10.1101/2021.02.26.21252554

2. Voloch CM, da Silva Francisco R, de Almeida LGP, et al (2021) Genomic characterization of a novel SARS-CoV-2 lineage from Rio de Janeiro, Brazil. J Virol. https://doi.org/10.1128/jvi.00119-21

3. Resende PC, Gräf T, Paixão ACD, et al (2021) A potential SARS-CoV-2 variant of interest (VOI) harboring mutation E484K in the Spike protein was identified within lineage B.1.1.33 circulating in Brazil. bioRxiv. https://doi.org/10.1101/2021.03.12.434969

4. Resende PC, Gräf T, Neto LG, et al. (2020) Identification of a new B.1.1.33 SARS-CoV-2 Variant of Interest (VOI) circulating in Brazil with mutation E484K and multiple deletions in the amino (N)terminal domain of the Spike protein. Virological.org 
5. Slavov SN, Leister Patané JS, Santos Bezerra R dos, et al (2021) Genomic monitoring unveil the early detection of the SARS-CoV-2 B.1.351 lineage (20H/501Y.V2) in Brazil. medRxiv. https://doi.org/10.1101/2021.03.30.21254591

6. Fujino T, Nomoto $H$, Kutsuna S, et al (2021) Novel SARS-CoV-2 Variant in Travelers from Brazil to Japan. Emerg Infect Dis 27:1243-1245. https://doi.org/10.3201/eid2704.210138

7. Cele S, Gazy I, Jackson L, et al (2021) Escape of SARS-CoV-2 501Y.V2 from neutralization by convalescent plasma. medRxiv. https://doi.org/10.1038/s41586-021-03471-w

8. Naveca F, da Costa C, Nascimento V, et al (2021) SARS-CoV-2 reinfection by the new Variant of Concern (VOC) P.1 in Amazonas, Brazil - SARS-CoV-2 coronavirus. In: Virological.org.

9. Faria N, Mellan TA, Whittaker C, et al (2021) Genomics and epidemiology of the P.1 SARS-CoV-2 lineage in Manaus, Brazil. Science. https://doi.org/10.1126/science.abh2644

10. Corman VM, Landt O, Kaiser M, et al (2020) Detection of 2019 -nCoV by RT-PCR. Euro Surveill 25:1-8 https://doi.org/10.2807/1560-7917.ES.2020.25.3.2000045

11. Naveca F, Nascimento V, Souza V, et al (2021) COVID-19 epidemic in the Brazilian state of Amazonas was driven by long-term persistence of endemic SARS-CoV-2 lineages and the recent emergence of the new Variant of Concern P.1. Nat Portf. https://doi.org/10.21203/rs.3.rs-275494/v1

12. Rambaut A, Holmes EC, O'Toole Á, et al (2020) A dynamic nomenclature proposal for SARS-CoV-2 lineages to assist genomic epidemiology. Nat Microbiol 5:1403-1407.

https://doi.org/10.1038/s41564-020-0770-5

13. Nguyen L-T, Schmidt HA, von Haeseler A, Minh BQ (2015) IQ-TREE: A Fast and Effective Stochastic Algorithm for Estimating Maximum-Likelihood Phylogenies. Mol Biol Evol 32:268-274. https://doi.org/10.1093/molbev/msu300

14. Amorim M, Souza W, Barros ACG, et al (2021) Respiratory Viral Shedding in Healthcare Workers Reinfected with SARS-CoV-2, Brazil, 2020. Emerg Infect Dis J 27:. https://doi.org/10.3201/eid2706.210558

15. Harrington D, Kele B, Pereira S, et al (2021) Confirmed Reinfection With Severe Acute Respiratory Syndrome Coronavirus 2 (SARS-CoV-2) Variant VOC-202012/01. Clin Infect Dis. https://doi.org/10.1093/cid/ciab014

16. Babiker A, Marvil CE, Waggoner JJ, et al (2020) The Importance and Challenges of Identifying SARSCoV-2 Reinfections. J Clin Microbiol 59. https://doi.org/10.1128/jcm.02769-20

17. Tillett RL, Sevinsky JR, Hartley PD, et al (2020) Genomic evidence for reinfection with SARS-CoV-2: a case study. Lancet Infect Dis 21:52-58. https://doi.org/10.1016/S1473-3099(20)30764-7 
18. Martins AF, Zavascki AP, Wink PL, et al (2021) Detection of SARS-CoV-2 lineage P.1 in patients from a region with exponentially increasing hospitalisation rate, February 2021, Rio Grande do Sul, Southern Brazil. Eurosurveillance 26:2100276. https://doi.org/10.2807/1560-7917.ES.2021.26.12.2100276

\section{Figures}

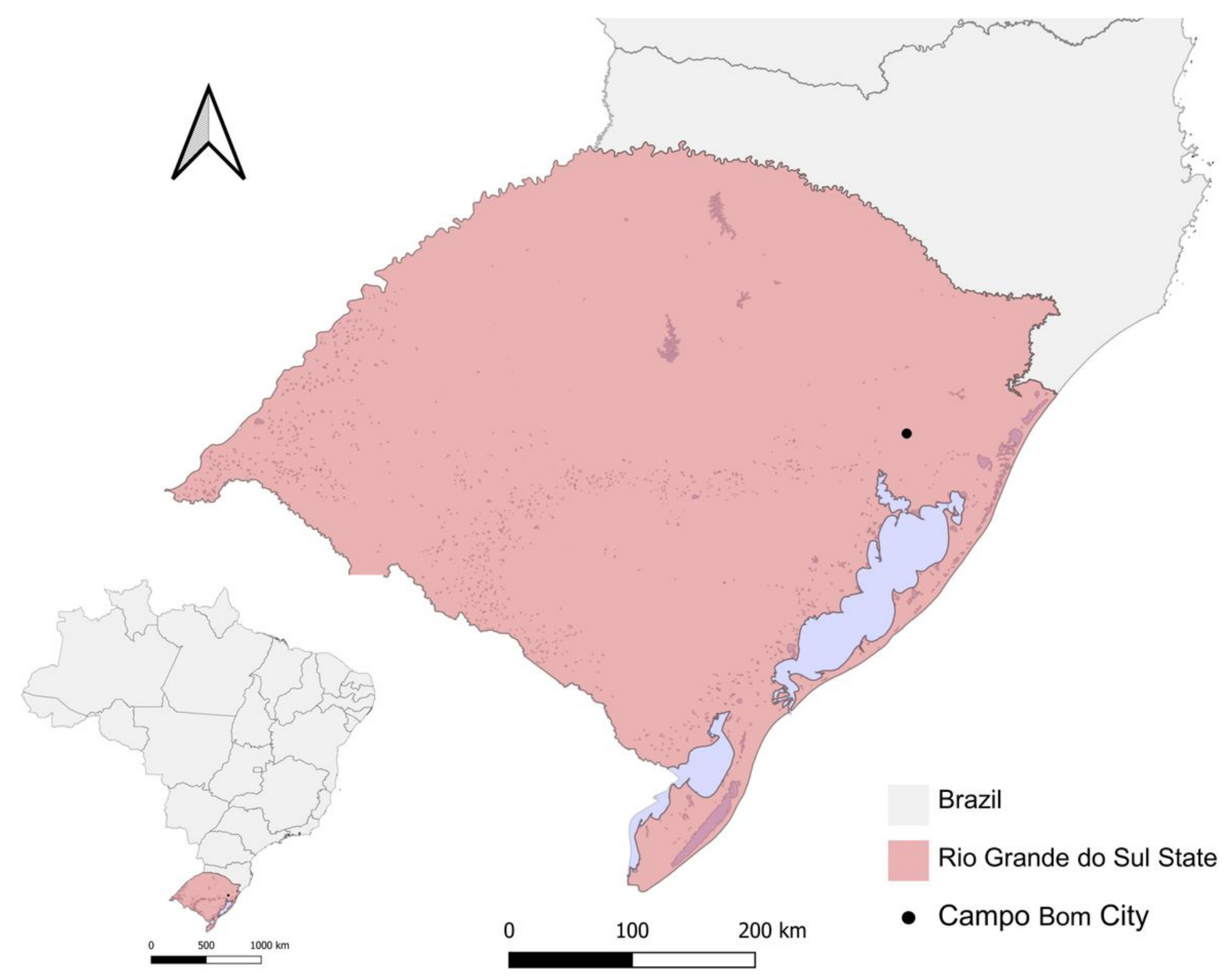

\section{Figure 1}

Reinfection case geographical location. On the left of the figure in minor scale the Brazil map and in pink the Rio Grande do Sul State of Brazil. The black dot represents Campo Bom city where the reinfection occurred, located in the northeastern region of the State. 
a.

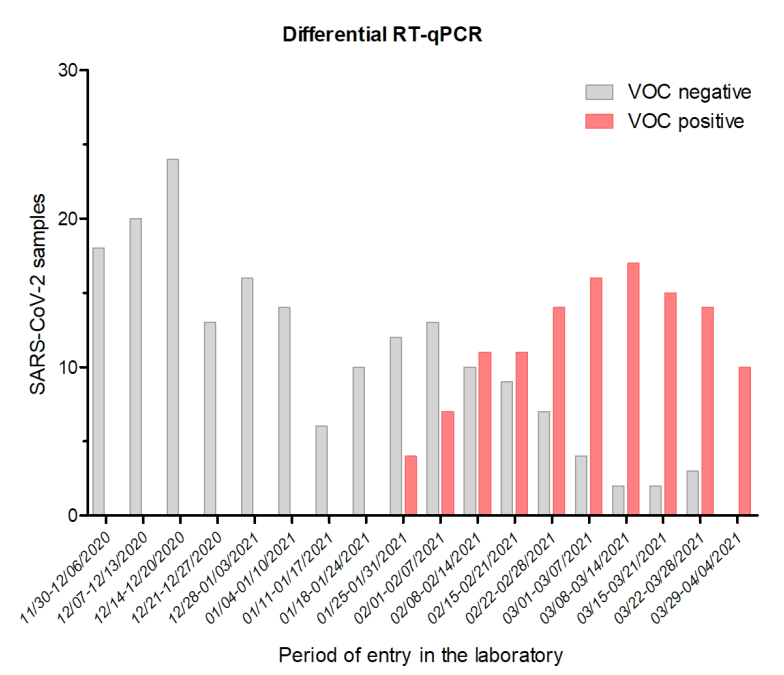

c.

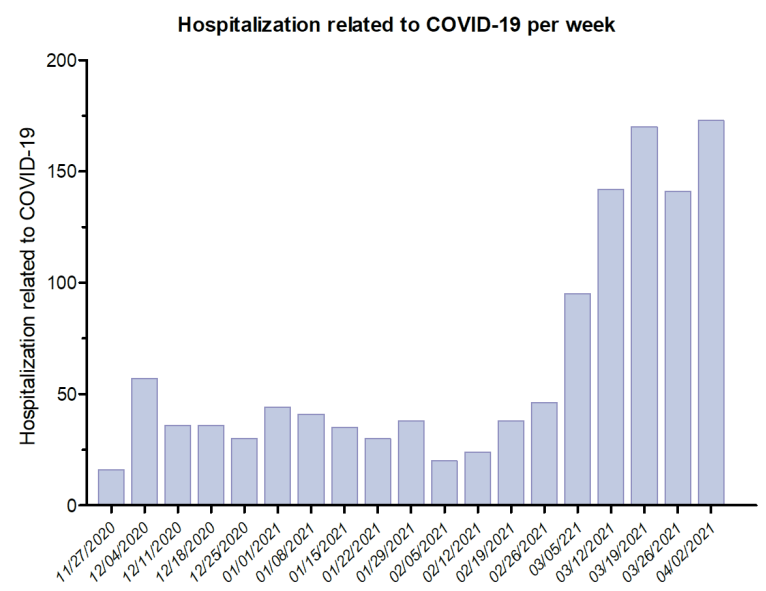

Date of publication by covid.saude.rs.gov.br b.

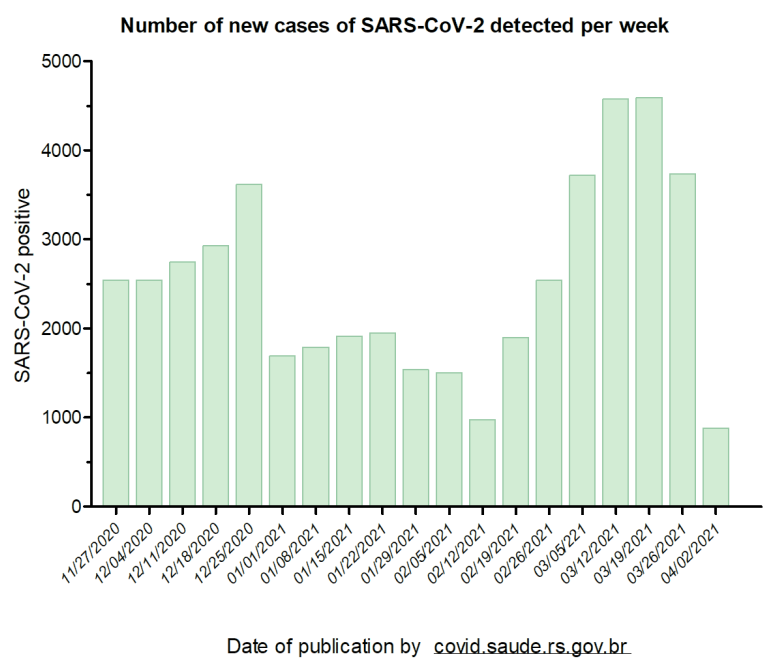

d.

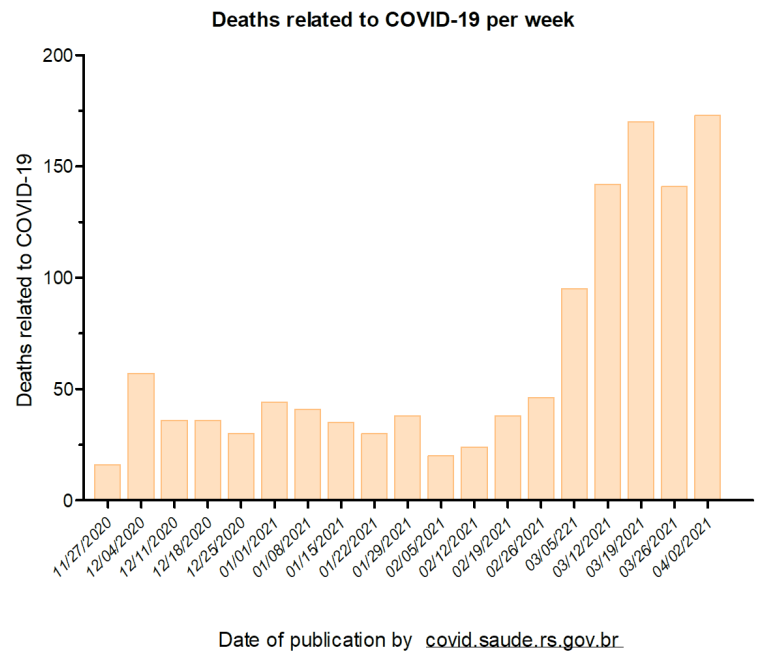

\section{Figure 2}

SARS-CoV-2 P.1 lineage emergence and COVID-19 general situation. (a) Retrospective analysis based on differential RT-qPCR: The gray bars represent the negative results predominating during the first epidemiological weeks. Afterwards, positive samples (represented by pink bars) replace the parental lines. (b) COVID-19 new cases (c) COVID-19 hospitalizations and (d) COVID-19 deaths. 


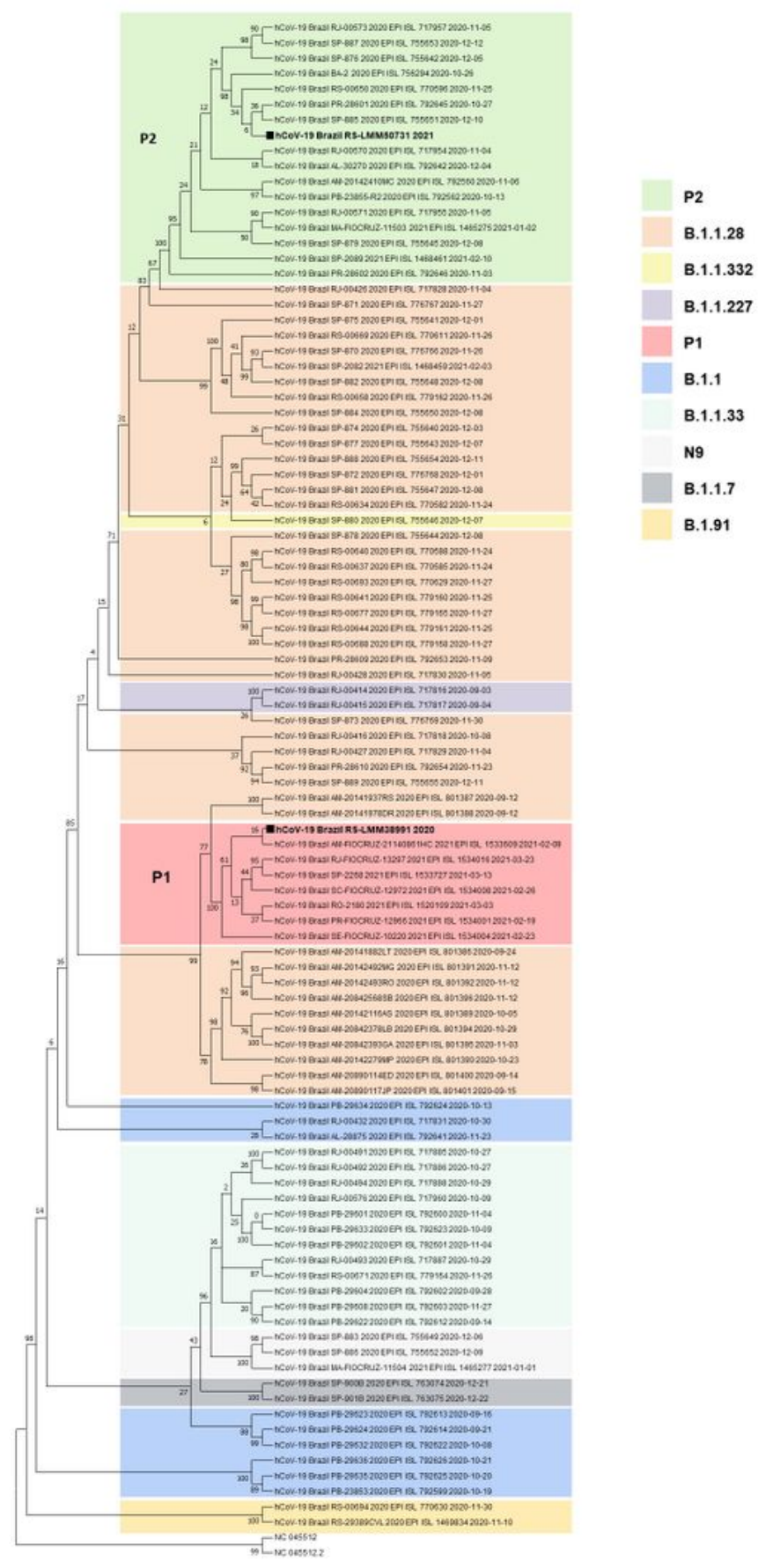

\section{Figure 3}

SARS-CoV-2 complete genome phylogenetic tree. The Maximum Likelihood phylogenetic analysis under General Time Reversible allowing for a proportion of invariable sites and with empirical and the substitution rates were inferred empirically in IQ-TREE v2.1.2 web server applying 200 replicates and 1000 bootstrap. 


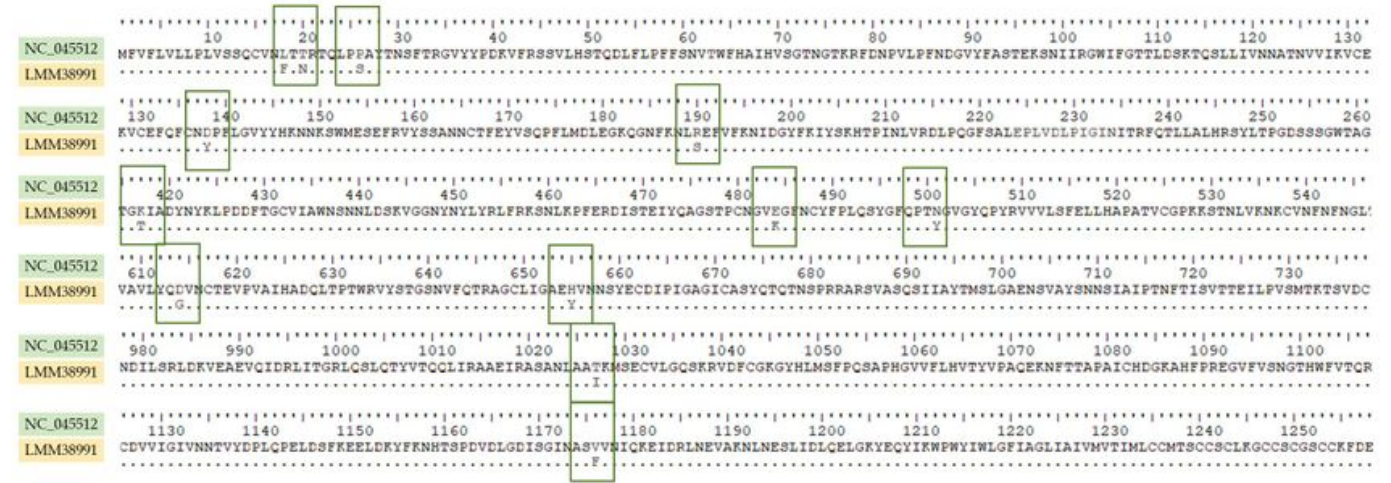

L18F - T20N - P26S - D192Y - R190S - K417T - E484K - N501Y -D614G - H655Y - T10211 - V1176F

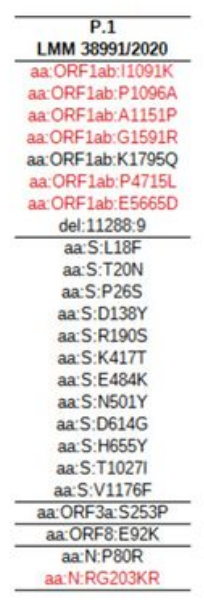

P.2 LMM38991

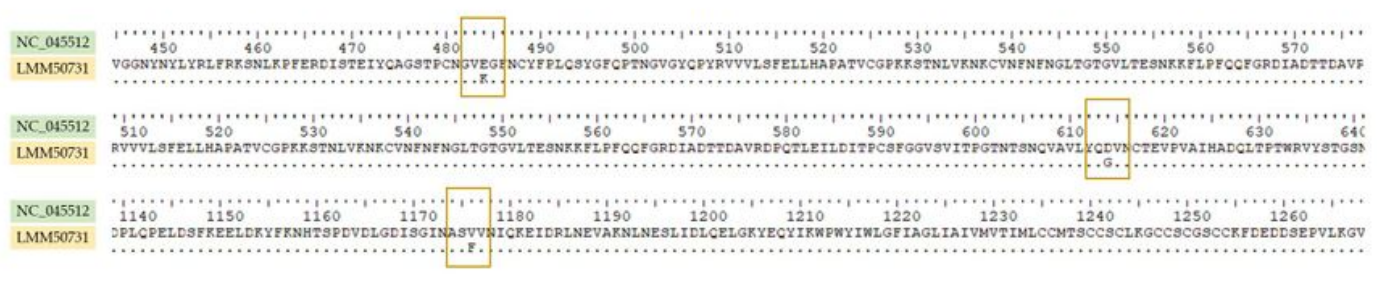

E484K - D614G -V1176F

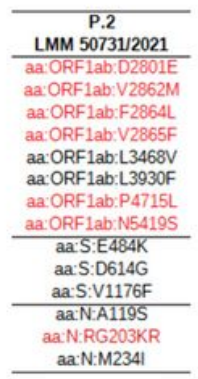

\section{Figure 4}

Amino acid mutations and INDELs. Spike amino acid alignment showing P.1 (above) and P.2 (below) mutations of the sequences obtained in this study. P.1 (L18F - T20N - P26S - D192Y - R190S - K417T E484K - N501Y -D614G-H655Y - T1021I - V1176F) and P.2 (E484K - D614G - V1176F).

\section{Supplementary Files}

This is a list of supplementary files associated with this preprint. Click to download.

- GisaidAcknowledgments.pdf 